\title{
Heart rate variability and baroreflex sensitivity in idiopathic dilated cardiomyopathy
}

\author{
J Hoffmann, W Grimm, V Menz, H-H Müller, B Maisch
}

\begin{abstract}
Objective-To examine the relation between cardiac autonomic tone, assessed by baroreflex sensitivity and heart rate variability, and left ventricular function, arrhythmias on Holter monitoring, and clinical variables in patients with idiopathic dilated cardiomyopathy.

Design-A prospective observational study.

Patients-160 patients with idiopathic dilated cardiomyopathy and preserved sinus rhythm in the absence of antiarrhythmic drug treatment. Measures of heart rate variability obtained by digital 24 hour Holter recordings included the mean of all coupling intervals between normal beats (RRm), the standard deviation of the mean of normal RR intervals (SDNN), and the square root of the mean of the squared differences between adjacent normal RR intervals (rMSSD). Baroreflex sensitivity testing was performed using the phenylephrine method.

Results-Mean SDNN (SEM) was 112 (46) ms, and baroreflex sensitivity was 7.5 (5.0) $\mathrm{ms} / \mathrm{mm} \mathrm{Hg}$. SDNN showed a weak correlation with baroreflex sensitivity $(r=0.19, \mathrm{p}<0.05)$ and with left ventricular ejection fraction $(r=0.29, \mathrm{p}<0.05)$. SDNN showed no significant correlation with age $(r=-0.07)$, the presence of non-sustained ventricular tachycardia $(r=-0.13)$, or left ventricular end diastolic diameter $(r=-0.07)$. In addition, baroreflex sensitivity showed no significant correlation with age $(r=-0.13)$, non-sustained ventricular tachycardia $(r=-0.08)$, left ventricular end diastolic diameter $(r=0.09)$, or ejection fraction $(r=0.14)$.

Conclusions-The weak correlation between baroreflex sensitivity and heart rate variability suggests that these two indices explore different aspects of cardiac autonomic control in patients with idiopathic dilated cardiomyopathy. The weak or absent correlation between baroreflex sensitivity, heart rate variability, and other potential non-invasive risk predictors, including left ventricular ejection fraction, left ventricular end diastolic diameter, and non-sustained ventricular tachycardia on Holter monitoring, indicate that these variables may have independent prognostic value in idiopathic dilated cardiomyopathy.
\end{abstract}

(Heart 2000;83:531-536)

Keywords: cardiomyopathy; baroreflex sensitivity; heart rate variability

The prognosis of patients with idiopathic dilated cardiomyopathy is poor, with annual death rates reported between $5 \%$ and $45 \% .^{1-4}$ Although little is known about the anatomical arrhythmogenic substrate in this disorder or about the cause of death, there is a large body of clinical evidence suggesting that approximately half of these patients die suddenly from ventricular tachyarrhythmias. ${ }^{56}$ Identification of patients who are at high risk of experiencing future cardiac events remains a major clinical problem. Imbalances in autonomic nervous system function have been shown to play a critical role in the genesis and maintenance of malignant tachyarrhythmias. $^{7-10}$ Both experimental and clinical studies have shown that increased sympathetic activity predisposes to malignant tachyarrhythmias in coronary disease, while parasympathetic activity increases the fibrillation threshold and exerts a protective effect. ${ }^{11-13}$ Over the last decade, two different non-invasive tests have become readily available for the quantitative assessment of the autonomic control of heart rate. The first is the determination of heart rate variability, which predominantly reflects tonic vagal activity ${ }^{14}{ }^{15}$; the second is the examination of baroreflex sensitivity as a measure of reflex autonomic response. ${ }^{16}{ }^{17}$

Recently, it has been reported that decreased baroreflex sensitivity and reduced heart rate variability are associated with an adverse prog- nosis in patients after myocardial infarction. ${ }^{18-21}$ In contrast to a large body of knowledge about vagal influences in ischaemic heart disease, only limited data are available on cardiac autonomic tone in patients with idiopathic dilated cardiomyopathy. To date, the only established risk predictor for overall mortality and sudden death in this disease remains the severity of left ventricular dysfunction. ${ }^{62}$ Because of this, the Marburg cardiomyopathy study (MACAS) was initiated in 1996 as a prospective observational study to determine the prognostic value of currently available non-invasive arrhythmia risk predictors in a large patient population with idiopathic dilated cardiomyopathy over a five year follow up. ${ }^{23}$ Our purpose in the present study was to determine the relation between autonomic dysfunction, as indexed by changes of heart rate variability and baroreflex sensitivity, and conventional arrhythmia risk predictors, including left ventricular dysfunction and arrhythmias on Holter monitoring, in the first 160 patients enrolled in MACAS.

\section{Methods}

PATIENTS

The study population consisted of 160 patients with idiopathic dilated cardiomyopathy enrolled in MACAS between 1996 and 1999. The protocol of MACAS has been described in detail elsewhere. ${ }^{23}$ Briefly, all patients met the

\footnotetext{
Dr Hoffmann.

email: HoMarburg@aol.com

Accepted 8 February 2000

Department of

Philipps-University of

Marburg

H-H Müller
} 
following inclusion criteria: age between 16 and 70 years, sinus rhythm in the absence of antiarrhythmic drugs including $\beta$ blockers, left ventricular enlargement, left ventricular ejection fraction $\leqslant 45 \%$ by both angiography and echocardiography, no history of myocardial infarction and no stenosis $>50 \%$ of any coronary artery by coronary angiography, and absence of valvar disease, systemic hypertension, or other conditions known to cause cardiomyopathy. ${ }^{24}$ In addition to complete diagnostic left and right heart catheterisation, endomyocardial biopsy was performed in 144 of the 160 study patients $(90 \%)$. This showed no evidence of acute myocarditis, specific heart muscle disease, or myocardial storage disease in any study patient. All patients were in a clinically stable condition with mild to moderate limitation of their daily activities, categorised as grade I to III of the New York Heart Association (NYHA) classification at study entry. Patients with a history of sustained ventricular tachyarrhythmias, congestive heart failure NYHA functional class IV, cancer, renal failure, severe liver disease, diabetes mellitus, clinical evidence of autonomic neuropathy, thyroid gland disease, or history of alcohol abuse were excluded from study participation. Furthermore, patients with atrial tachycardia, sinus node disease, second or third degree atrioventricular block, and pacemaker dependent patients were also excluded from the study.

HOLTER MONITORING

Ambulatory Holter ECG recordings were performed in all 160 study patients while they were off antiarrhythmic drug treatment, using an Oxford two channel or three channel digital recorder (Oxford Instruments, Abingdon, UK; range 20-24 hours) with manual overread by an experienced nurse. The number of ventricular premature beats during each hour was assessed, and the mean value calculated by using the Oxford Medilog Excel 2 system (version 7.5, Oxford Instruments). Holter recordings were further categorised according to the presence of more than 10 ventricular premature beats/hour and according to the presence or absence of non-sustained ventricular tachycardia. Non-sustained ventricular tachycardia was defined as $\geqslant 3$ consecutive ventricular premature beats with a rate $\geqslant 120$ beats $/ \mathrm{min}$ lasting $<30$ seconds detected on the same Holter recordings that were used to determine heart rate variability measurements.

ANALYSIS OF HEART RATE VARIABILITY

Heart rate variability was computed from the same digital recordings that were used to evaluate the presence or absence of rhythm disorders. At least 20 hours of artefact-free recording time was required for patients to be eligible for the present study. Otherwise, the Holter ECG was discarded, and a new Holter was started. Data were analysed by the Oxford ${ }^{\mathbb{B}}$ Medilog Excel 2 system. Only RR cycles in which beats had a normal morphology and where cycle lengths were within $25 \%$ of the duration of the preceding cycle length were included for heart rate variability analysis, in order to ensure abolition of ectopic beats and artefacts. When sinus rhythm was interrupted by a premature atrial or ventricular beat, one RR interval preceding and one following the non-sinus beat were rejected, and the ectopic beat was substituted by computing the average of neighbouring normal RR intervals. After this labelling process was finished, the data file was manually overread and corrected where appropriate. For each Holter recording, the mean of all coupling intervals between normal beats (RRm) was calculated, and the following standard time domain parameters were determined: (1) the standard deviation of all normal $\mathrm{RR}$ intervals (SDNN); and (2) the square root of the mean of the squared differences between adjacent normal RR intervals (rMSSD) over the whole recording period.

MEASUREMENT OF BAROREFLEX SENSITIVITY After informed consent was obtained, baroreflex sensitivity was performed in 160 study patients by intravenous administration of phenylephrine, according to a method described by Smyth and coworkers. ${ }^{16}$ All patients were studied in a fasting state and supine, between 9 am and 12 noon. A slow intravenous saline infusion was started. Arterial pressure curves and beat to beat intervals were continuously monitored noninvasively, using the infrared digital plethysmographic technique (Finapres 2300 system, Ohmeda, Hatfield, UK). Initially, a test bolus of the $\alpha_{1}$ agonist phenylephrine $(2 \mu \mathrm{g} / \mathrm{kg})$ was rapidly injected to evaluate the magnitude of the resulting blood pressure increase. If systolic blood pressure did not increase as desired $(>15$ $\mathrm{mm} \mathrm{Hg}$ ), additional injections were made at intervals of 10 minutes increasing the phenylephrine dose up to a maximum of $10 \mu \mathrm{g} / \mathrm{kg}$. Phenylephrine injections were repeated until at least two recordings were obtained using the optimal bolus dose. All blood pressure data and corresponding RR interval data were transferred online from the Finapres 2300 system to an IBM personal computer using a custom written software program. Data from these files were directly imported into a commercially available computer program (MS Excel version 5.0 for Windows 3.11) for further analysis. Using this software, a linear regression analysis of $R R$ cycles and systolic blood pressure values was performed, including all values between the beginning and end of the blood pressure increase. Baroreflex sensitivity was then calculated as the slope of the linear regression line relating changes in RR intervals to systolic blood pressure changes $(\mathrm{ms} / \mathrm{mm} \mathrm{Hg})$. As in the ATRAMI study (automatic tone and reflexes after myocardial infarction), ${ }^{21}$ only regression lines with significant correlation coefficients $(p<0.05)$ were accepted for baroreflex sensitivity analysis.

\section{STATISTICAL ANALYSIS}

Values are presented as mean (SD). Regression lines for baroreflex sensitivity were calculated with the least squares fit method. Pearson's product-moment correlation coefficient $(r)$ was employed to test the strength of linear association between variables. Correlation coefficients 
Table 1 Characteristics of 160 study patients with idiopathic dilated cardiomyopathy

\begin{tabular}{ll}
\hline Clinical characteristics & $n(\%)^{\star}$ \\
\hline Women & $40(25)$ \\
Men & $120(75)$ \\
Mean (SD) age (years) & $48(12)$ \\
$\quad$ Range & 17 to 69 \\
NYHA classification & $26(16)$ \\
Class I & $88(55)$ \\
Class II & $46(29)$ \\
Class III & $31(10)$ \\
Echocardiographic study & 15 to 45 \\
Mean (SD) LVEF (\%) & $68(8)$ \\
Range & 57 to 90 \\
Mean (SD) LVEDD (mm) & $123(77)$ \\
Range & $119(74)$ \\
Drug treatment for heart failure & $131(82)$ \\
$\quad$ Digoxin &
\end{tabular}

$\star$ Unless otherwise stated.

ACE, angiotensin converting enzyme; LVEDD, left ventricular end diastolic diameter; LVEF, left ventricular ejection fraction.

were tested for significance using the $t$ test. A value of $p<0.05$ was considered significant.

In addition, multivariate linear regression analysis was performed using the "REG procedure" of the statistical software package SAS version 6.12. The best multivariate model for baroreflex sensitivity and SDNN was selected using Akaike's information criterion. ${ }^{25}$

\section{Results}

The clinical characteristics of the 160 study patients with idiopathic dilated cardiomyopathy are summarised in table 1 . There were 120 men, mean age was 48 (12) years, and mean left ventricular ejection fraction was $31(10) \%$. All patients were in clinically stable condition at the time of study entry on standard heart failure treatment comprising digitalis, diuretics, and an angiotensin converting enzyme inhibitor in the vast majority of study patients (table 1 ).

RELATION BETWEEN HEART RATE VARIABILITY, BAROREFLEX SENSITIVITY, CLINICAL CHARACTERISTICS, ARRHYTHMIAS, AND LEFT VENTRICULAR DYSFUNCTION

Whereas heart rate variability analysis could be performed in all 160 patients with idiopathic dilated cardiomyopathy, an acceptable baroreflex sensitivity test result, as defined above, could only be obtained in 137 of the patients $(86 \%)$. Baroreflex sensitivity testing did not show a significant correlation between RR interval changes and systolic blood pressure

Table 2 Correlations between heart rate variability, baroreflex sensitivity, and clinical and echocardiographic variables

\begin{tabular}{|c|c|c|c|c|c|c|c|c|}
\hline & Age & NYHA & LVEF & LVEDD & NSVT+ & $R R m$ & $S D N N$ & $r M S S D$ \\
\hline \multicolumn{9}{|l|}{ Age } \\
\hline NYHA & 0.08 & & & & & & & \\
\hline LVEF & -0.05 & -0.45 & & & & & & \\
\hline LVEDD & 0.03 & 0.21 & $-0.51^{\star \star}$ & & & & & \\
\hline NSVT+ & 0.12 & $0.21^{\star \star}$ & $-0.23^{\star}$ & $0.23^{\star \star}$ & & & & \\
\hline $\mathrm{RRm}$ & 0.06 & -0.16 & $0.23^{\star}$ & -0.03 & -0.04 & & & \\
\hline SDNN & -0.07 & $-0.19^{\star}$ & $0.29^{\star \star}$ & -0.07 & -0.13 & $0.69^{\star \star}$ & & \\
\hline RMSSD & 0.08 & -0.09 & 0.08 & 0.08 & 0.05 & $0.52^{\star \star}$ & $0.58^{\star \star}$ & \\
\hline BRS & -0.13 & $-0.22^{\star}$ & 0.14 & 0.09 & -0.08 & 0.09 & $0.19^{\star}$ & -0.01 \\
\hline
\end{tabular}

${ }^{\star} \mathrm{p}<0.05 ;{ }^{\star \star} \mathrm{p}<0.01$.

BRS, baroreflex sensitivity; LVEDD, left ventricular end diastolic diameter; LVEF, left ventricular ejection fraction; NSVT+, presence of non-sustained ventricular tachycardia; RRm, the mean of all coupling intervals between normal beats; rMSSD, the square root of the mean of the squared differences between adjacent normal RR intervals; SDNN, the standard deviation of all normal $\mathrm{RR}$ intervals.

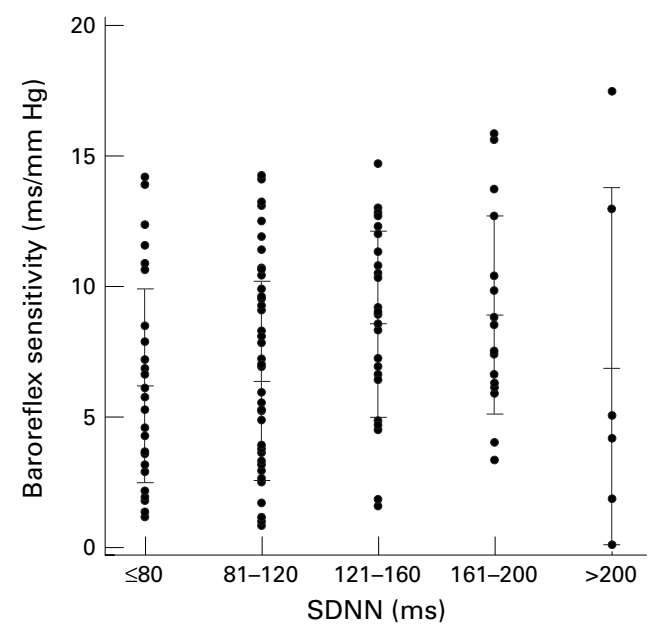

Figure 1 Plot of the mean and SD of baroreflex sensitivity for SDNN bins. The correlation coefficient between these variables was only $0.19(p<0.05)$.

increase in 21 patients owing to frequent premature beats. In the remaining two patients, no adequate increase in blood pressure of at least $15 \mathrm{~mm} \mathrm{Hg}$ could be achieved with the maximum dose level of phenylephrine applied in the present study $(10 \mu \mathrm{g} / \mathrm{kg}$ body weight).

Mean SDNN (SEM) was 112 (46) $\mathrm{ms}$, and baroreflex sensitivity was $7.5(5.0) \mathrm{ms} / \mathrm{mm} \mathrm{Hg}$. The relation between measures of heart rate variability and baroreflex sensitivity is summarised in table 2. SDNN as an index of overall variability showed only a weak correlation with baroreflex sensitivity $(r=0.19, \mathrm{p}<0.05)$ (fig 1). In addition, there was only a weak correlation between SDNN and the left ventricular ejection fraction $(r=0.29, \mathrm{p}<0.05)$ (fig 2$)$. rMSSD, reflecting predominantly parasympathetic activity, was not correlated with left ventricular dysfunction $(r=0.08)$. SDNN showed no significant correlation with age $(r=-0.07)$, the presence of non-sustained ventricular tachycardia on Holter $(r=-0.13)$, or left ventricular end diastolic diameter $(r=-0.07)$.

Baroreflex sensitivity showed no significant correlation with age $(r=-0.13)$, the presence of spontaneous non-sustained ventricular

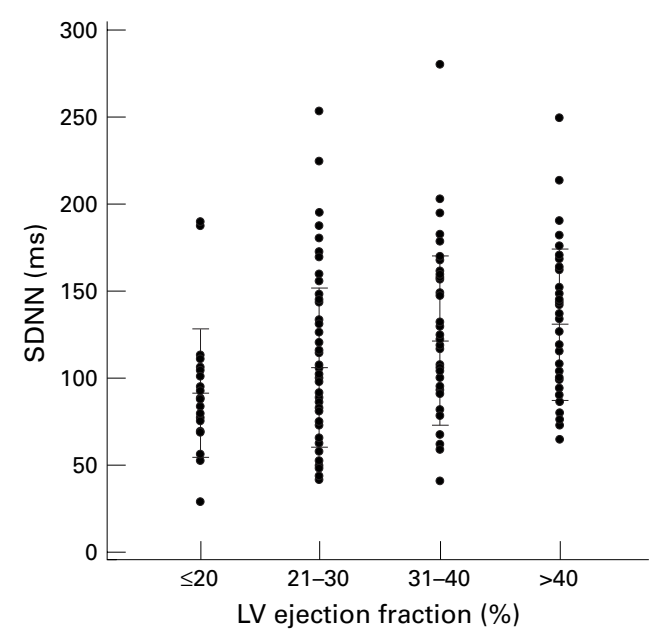

Figure 2 Plot of the mean and SD of SDNN for left ventricular ejection fraction bins showing only a moderate correlation between these variables $(r=0.29, p<0.05)$. 


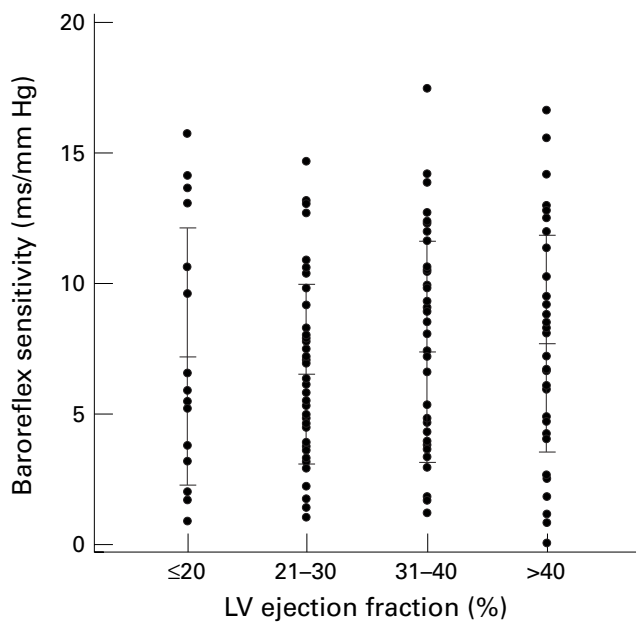

Figure 3 Plot of the mean and SD of baroreflex sensitivity for left ventricular ejection fraction bins showing no significant correlation between these variables $(r=0.14)$.

tachycardia $(r=-0.08)$, left ventricular end diastolic diameter $(r=0.09)$, or left ventricular ejection fraction $(r=0.14$ ) (fig 3). Figure 4 shows that there was no significant association between heart rate variability, baroreflex sensitivity and the presence of non-sustained ventricular tachycardia on Holter monitoring.
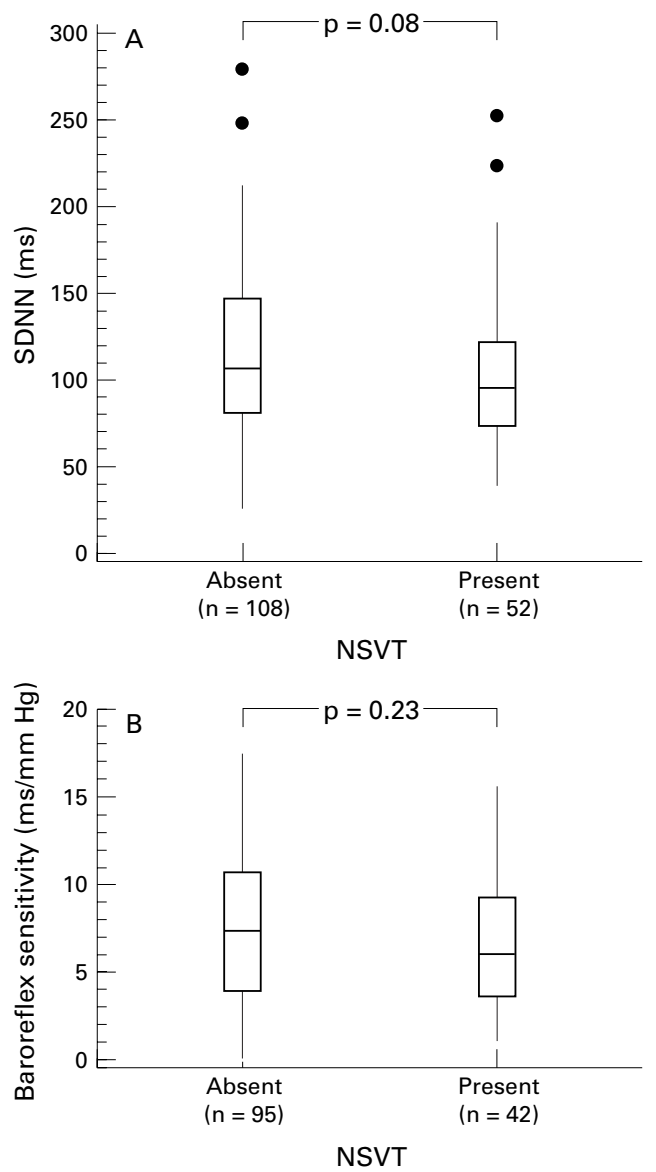

Figure 4 (A) SDNN and (B) baroreflex sensitivity according to the presence or absence of non-sustained ventricular tachycardia (NSVT) on Holter monitoring. There was no significant difference in heart rate variability and baroreceptor sensitivity between patients with and without NSVT.
MULTIVARIATE MODEL FOR SDNN AND BAROREFLEX SENSITIVITY

Multivariate linear regression analysis revealed the following model for baroreflex sensitivity:

Baroreflex sensitivity $=1.27-1.81 \times \mathrm{NYHA}+$ $0.116 \times \mathrm{LVEDD}+0.0268 \times \mathrm{SDNN}-0.0245$ $\times$ rM SSD,

where LVEDD is left ventricular end diastolic dimension.

The measure of determination $R^{2}$ for this model was only $11 \%$-that is, only $11 \%$ of the baroreflex sensitivity variance can be explained by the best multivariate linear regression model. For the heart rate variability variable SDNN, multivariate regression analysis resulted in the following model:

$\mathrm{SDNN}=-24.86+0.367 \times \mathrm{rMSSD}-0.302 \times$ age $-12.35 \times \mathrm{NSVT}+0.174 \times \mathrm{RRm}+0.982$ $\times \mathrm{BRS}$,

where BRS is baroreflex sensitivity and NSVT is non-sustained ventricular tachycardia.

The measure of determination $R^{2}$ for this model was $54 \%$ - that is, $54 \%$ of the heart rate variability variable SDNN can be explained by the best multivariate linear regression model.

\section{Discussion}

In this study we investigated the relation between heart rate variability, baroreflex sensitivity, clinical characteristics, arrhythmias on Holter, left ventricular size, and left ventricular ejection fraction in the first 160 consecutive patients enrolled in the Marburg cardiomyopathy study (MACAS) ${ }^{23}$ We found only a weak correlation between baroreflex sensitivity and heart rate variability in this homogeneous group of patients with idiopathic dilated cardiomyopathy, suggesting that these two variables explore different aspects of the autonomic control of the heart. In addition, the weak or absent correlation between baroreflex sensitivity, heart rate variability, and other non-invasive potential risk predictorsincluding left ventricular ejection fraction, left ventricular end diastolic diameter, and arrhythmias on Holter monitoring - indicates that these variables may have independent prognostic value in this disease. The prognostic significance of these findings will be determined by multivariate Cox analysis after a five year follow up of at least 200 patients in MACAS.

ASSESSMENT OF CARDIAC AUTONOMIC TONE USING HEART RATE VARIABILITY AND BAROREFLEX SENSITIVITY TESTING

Numerous clinical and experimental studies have shown a link between autonomic imbalance and cardiovascular mortality, including sudden death. ${ }^{7-21}$ With growing awareness of the key role of neural mechanisms in arrhythmogenesis, attention has been focused on the evaluation of the autonomic system by using non-invasive tests of autonomic function such as heart rate variability analysis and baroreflex sensitivity testing. While heart rate variability predominantly reflects tonic vagal activity, baroreflex sensitivity is widely used to determine reflex vagal activity in patients after myocardial infarction. ${ }^{19-2126}$ Both measures may help provide insight into complex physiological and pathological processes and so 
enhance risk stratification for arrhythmic events and mortality in patients with coronary artery disease and dilated cardiomyopathy.

COMPARISON WITH PREVIOUS STUDIES IN DILATED CARDIOMYOPATHY

The present study differs from previous reports in several important aspects. In contrast to all previously published studies on idiopathic dilated cardiomyopathy, we investigated the correlation between both baroreflex sensitivity and heart rate variability and several parameters of left ventricular function, including left ventricular ejection fraction and left ventricular end diastolic diameter. Our finding that heart rate variability, baroreflex sensitivity, and left ventricular ejection fraction had only a weak to moderate correlation with each other suggests that they may be of independent prognostic value in patients suffering from idiopathic dilated cardiomyopathy. In contrast to many previous studies, our study investigated a relatively homogeneous group of patients with this disease, all of whom had previously undergone left and right heart catheterisation, including endomyocardial biopsy in $90 \%$. It should be noted that patients on antiarrhythmic drug treatment that could not be discontinued for at least five half lives, as well as those on amiodarone and $\beta$ blockers, were excluded from participation in the study. Both antiarrhythmic drugs and $\beta$ blockers have previously been shown to alter heart rate variability and baroreflex sensitivity. ${ }^{27}$

In a previous smaller study at our institution, which involved 71 patients with idiopathic dilated cardiomyopathy, heart rate variability analysis was not found to be a valuable tool for predicting major arrhythmic events, including sudden death. ${ }^{28}$ In contrast to MACAS, however, the study population and the follow up duration of our previous study were insufficient for us to exclude with confidence a minor to moderate role of heart rate variability in arrhythmia risk prediction. Also, baroreflex sensitivity testing was not performed in that study.

Several other investigators have examined the prognostic role of heart rate variability in patients with dilated cardiomyopathy ${ }^{29-31}$ and in mixed patient populations suffering from congestive heart failure. ${ }^{32-35}$ As in the present study, Fauchier and colleagues ${ }^{30}$ found a weak to moderate correlation between heart rate variability (SDNN) and left ventricular function assessed by shortening fraction $(r=0.5)$ in 93 patients with dilated cardiomyopathy. By multivariate analysis, these investigators identified SDNN as an independent predictor of cardiac death or heart transplant, in addition to left ventricular end diastolic diameter and increased pulmonary capillary wedge pressure. Yi and colleagues also showed that reduced heart rate variability had independent predictive value for death from progressive heart failure in a cohort of 64 patients with dilated cardiomyopathy. ${ }^{31}$ In a large prospective multicentre study, Nolan and colleagues investigated the prognostic value of heart rate variability in 433 outpatients with chronic heart failure. ${ }^{35}$ In that study, SDNN was associated with death from progressive heart failure but not with sudden cardiac death. Mortara and colleagues focused on the prognostic value of baroreflex sensitivity testing in 282 patients with chronic heart failure. ${ }^{36}$ They found a significantly higher mortality among patients with greatly reduced baroreflex sensitivity ( $<1.3 \mathrm{~ms} / \mathrm{mm} \mathrm{Hg}$ ) compared with those with more preserved reflex activity. However, half of the patients in that study were suffering from ischaemic heart disease.

\section{CLINICAL IMPLICATIONS}

Based on the findings of the present study as well as previous studies with smaller patient populations, both heart rate variability and baroreflex sensitivity may represent important prognostic markers in patients with idiopathic dilated cardiomyopathy, in addition to conventional variables such as left ventricular size, left ventricular ejection fraction, and arrhythmias on Holter monitoring. Until appropriately designed studies like MACAS are finished, not enough data will be available to justify any therapeutic action based on heart rate variability or baroreflex sensitivity tests - for example, prophylactic defibrillator implantation as an adjunct to optimised standard heart failure treatment in patients with idiopathic dilated cardiomyopathy.

This study was in part supported by a grant from the German Science Foundation, Bonn, Germany.

1 Abelman WH, Beverly HL. The challenge of cardiomyopathy. F Am Coll Cardiol 1989;13:1219-39.

2 Anderson KP, Freedman RA, Maron JW. Sudden death in idiopathic dilated cardiomyopathy. Ann Intern Med 1987; 107:104-6.

3 Fuster V, Gersh BJ, Giuliani ER, et al. The natural history of idiopathic dilated cardiomyopathy. Am 7 Cardiol 1981;47: 525-31

4 Dec GW, Fuster V. Idiopathic dilated cardiomyopathy. $N$ Engl f Med 1994;331:1565-75.

5 Bigger JT. Why patients with congestive heart failure die. Arrhythmias and sudden cardiac death. Circulation 1987;

75(suppl IV):IV-28-35.
6 Tamburro P, Wilber D. Sudden death in idiopathic dilated cardiomyopathy. Am Heart $\mathcal{F}$ 1992;124:1035-45.

7 Lown B, Verrier RL. Neural activity and ventricular fibrillaLown B, Verrier RL. Neural activity and
tion. N Engl f Med 1976;294:1165-70.

8 Malliani A, Schwartz PJ, Zanchetti A. Neural mechanisms in Malliani A, Schwartz PJ, Zanchetti A. Neural mechanisms in
life threatening arrhythmias. Am Heart $\mathcal{f}$ 1980;100:705-15.

life threatening arrhythmias. Am Heart f 1980;100:705-15.
Schwartz PJ, Stone HL. The role of the autonomic nervous system in sudden coronary death. Ann NY Acad Sci 1982; 382:162-80

10 Schwartz PJ, La Rovere MT, Vanoli E. Autonomic nervous system and sudden cardiac death. Experimental basis and clinical observations for post-myocardial infarction risk stratification. Circulation 1992;85(suppl I):I-77-91.

11 Vanoli E, De FGM, Stramba-Badiale M, et al. Vagal stimulation and prevention of sudden death in conscious dogs with a healed myocardial infarction. Circ Res 1991;68: 1471-81.

12 Hull SS, Evans AR, Vanoli E, et al. Heart rate variability before and after myocardial infarction in conscious dogs at high and low risk of sudden death. F Am Coll Cardiol 1990; 16:978-85.

13 Barron HV, Lesh MD. Autonomic nervous system and sudden cardiac death. F Am Coll Cardiol 1996;27:1053-60.

14 Akselrod S, Gordon U, Ubel FA, et al. Power spectrum analysis of heart rate fluctuation: a quantitative probe of sis of heart rate fluctuation: a quantitative probe of

15 Task Force of the European Society of Cardiology and the North American Society of Pacing and Electrophysiology. Heart rate variability. Standards of measurement, physiological interpretation, and clinical use. Circulation 1996; 93:1043-65.

16 Smyth HS, Sleight P, Pickering GW. Reflex regulation of arterial pressure during sleep in man: a quantitative method of assessing baroreflex sensitivity. Circ Res 1969;24:109-22.

17 Billman GE, Schwartz PJ, Stone HL. Baroreceptor reflex control of heart rate: a predictor of sudden cardiac death Circulation 1982;66:874-80.

18 Kleiger RE, Miller JP, Bigger JT, et al. Decreased heart rate variability and its association with increased mortality after acute myocardial infarction. Am f Cardiol 1987;59:256-62.

19 La Rovere MT, Specchia G, Mortara A, et al. Baroreflex sensitivity, clinical correlates, and cardiovascular mortality among patients with a first myocardial infarction: a prospective study. Circulation 1988;78:816-24. 
20 Farrell TG, Odemuyiwa O, Bashir Y, et al. Prognostic value of baroreflex sensitivity testing after acute myocardial of baroreflex sensitivity testing after
infarction. Br Heart f 1992;67:129-37.

21 La Rovere MT, Bigger JT, Marcus FI, et al. for the ATRAMI (Autonomic Tone and Reflexes After Myocardial Infarction) Investigators. Baroreflex sensitivity and heart-rate variability in prediction of total cardiac mortality after myocardial infarction. Lancet 1998;351:478-84.

22 Sugrue DD, Rodeheffer RJ, Codd MB, et al. The clinical course of idiopathic dilated cardiomyopathy: a populationbased study. Ann Intern Med 1992;117:117-23.

23 Grimm W, Glaveris C, Hoffmann J, et al. Noninvasive risk stratification in idiopathic dilated cardiomyopathy: design and first results of the Marburg Cardiomyopathy Study. PACE 1998;21(II):2551-6.

24 Richardson P, McKenna W, Bristow M, et al. Report of the 1995 World Health Organization International Society and Federation of Cardiology Task Force on the Definition and Classification of Cardiomyopathies. Circulation 1996;93: 841-2.

25 SAS/STAT user's guide, version 6, 4th ed, vol 2. Cary, North Carolina: SAS Institute Inc, 1989:1351-457.

26 Hohnloser $\mathrm{SH}$, Klingenheben $\mathrm{T}$, van de Loo A, et al. Reflex versus tonic vagal activity as a prognostic parameter in versus tonic vagal activity as a prognostic parameter in parients with sustained ventricular tachycardion. Circulation 1994;89:1068-73.

27 Zuanetti G, Latini R, Neilson JM, et al, and the Antiarrhythmic Drug Evaluation Group (ADEG). Heart rate variability in patients with ventricular arrhythmias: effect of antiarrhythmic drugs. $\mathcal{F}$ Am Coll Cardiol 1991;17:604-12.

28 Hoffmann J, Grimm W, Menz V, et al. Heart rate variability and major arrhythmic events in patients with idiopathic dilated cardiomyopathy. PACE 1996;19(II):1841-4.
29 Ajiki K, Murakawa Y, Yanagisawa-Miwa A, et al. Autonomic nervous system activity in idiopathic dilated cardiomyopathy and in hypertrophic cardiomyopathy. Am $\mathcal{F}$ Cardiol 1993; 71:1316-20.

30 Fauchier L, Babuty D, Cosnay P, et al. Heart rate variability in idiopathic dilated cardiomyopathy: characteristics and prognostic value. I Am Coll Cardiol 1997;30:1009-14.

31 Yi G, Goldman JH, Keeling PJ, et al. Heart rate variability in idiopathic dilated cardiomyopathy: relation to disease idiopathic dilated cardiomyopathy: relation to
severity and prognosis. Br Heart $\mathcal{F} 1997 ; 77: 108-14$.

32 Ponikowski P, Anker SD, Chua TP, et al. Depressed heart rate variability as an independent predictor of death in congestive heart failure secondary to ischemic or idiopathic dilated cardiomyopathy. Am f Cardiol 1997;79:1645-50.

33 Szabó BM, van Veldhuisen DJ, van der Veer N, et al. Prognostic value of heart rate variability in chronic heart failure secondary to idiopathic or ischemic dilated cardiomyopathy. Am f Cardiol 1997;79:978-80.

34 Brouwer J, van Veldhuisen DJ, Man in't Veld AJ, et al, for the Dutch Ibopamine Multicenter Trial Study Group. Prognostic value of heart rate variability during long-term follow-up in patients with mild to moderate heart failure. $\mathcal{F}$ Am Coll Cardiol 1996;28:1183-9.

35 Nolan J, Batin PD, Andrews R, et al. Prospective study of heart rate variability and mortality in chronic heart failure. Results of the United Kingdom Heart failure Evaluation and Assessment of Risk Trial (UK-Heart). Circulation 1998;98:1510-16.

36 Mortara A, La Rovere MT, Pinna GD, et al. Arterial baroreflex modulation of heart rate in chronic heart failure. Clinical and hemodynamic correlates and prognostic implications. Circulation 1997;96:3450-8.

\section{Electronic pages}

\section{eHEART: www.heartjnl.com}

The following electronic only articles were published in conjunction with the April issue of Heart.

\section{Angiographic documented coronary arterial spasm in absence of critical coronary artery stenoses in a patient with variant angina episodes during exercise and dobutamine stress echocardiography \\ M Roffi, B Meier, Y Allemann}

Dobutamine stress echocardiography is widely performed as a useful diagnostic tool in patients with known or suspected coronary artery disease. Dobutamine induced myocardial ischaemia is frequently associated with ST segment depression. ST segment elevation is uncommon and is almost always associated with prior myocardial infarction or transient total coronary occlusion. Dobutamine induced ST segment elevation in absence of significant coronary artery disease is a rare condition and is supposed to be a consequence of severe coronary artery spasm. The case of a 58 year old man with variant angina episodes at rest, during exercise test, and dobutamine stress echocardiography is reported, in whom coronary spasm without significant coronary artery stenoses was documented angiographically.

(Heart 2000;83:e4) www.heartjnl.com/cgi/content/full/83/4/e4

Acute severe thrombocytopenia after treatment with ReoPro (abciximab)

\section{$R$ Butler, P F B Hubner}

ReoPro (abciximab) is an extremely potent inhibitor of the glycoprotein IIb/IIIa receptor, the final common pathway of platelet activation and aggregation. Its main role is the maintenance of coronary patency after suboptimal results with coronary intervention. However, one of the complications of this treatment is excessive bleeding, a problem which may be compounded by a rare idiosyncratic thrombocytopenic reaction. A severe episode of thrombocytopenia in a 64 year old man is described; he was treated with ReoPro for a right coronary stenosis which had not been resolved by angioplasty. His platelet level dropped quickly and only improved after 20 units of platelets were given.

(Heart 2000;83:e5) www.heartjnl.com/cgi/content/full/83/4/e5

Cystic tumour of the atrioventricular nodal region: report of a case successfully treated with surgery

f R Paniaguaa, f R Sadabaa, L A Davidsonb, C M Munscha

A case is reported of a 59 year old woman who presented with palpitations. Electrocardiographic studies revealed atrial fibrillation and atrioventricular block. Echocardiography and magnetic resonance imaging showed a right atrial cystic mass attached to the interatrial septum. The patient underwent surgical excision of the mass. Histopathological findings were of a cystic tumour of the atrioventricular nodal region. This is the second report of this condition diagnosed antemortem and treated successfully with surgical excision.

(Heart 2000;83:e6) www.heartjnl.com/cgi/content/full/83/4/e6

Left ventricular opacification during selective intracoronary injection of echocardiographic contrast in patients with hypertrophic cardiomyopathy

$P M$ Elliott, $S$ f Brecker, Wf McKenna

Percutaneous alcohol ablation of the interventricular septum via the first septal perforator branch of the left anterior descending artery can successfully treat dynamic left ventricular outflow tract obstruction in patients with hypertrophic cardiomyopathy. Increasingly, echocardiographic contrast agents are used before alcohol injection to identify the perfusion bed of the septal perforator vessels. This study describes the unexpected opacification of the left ventricular cavity in three of five consecutive patients following selective injection of the first septal perforator with Optison. This case study demonstrates that direct communication between the first septal perforator vessel and the left ventricle is common, an observation that may have considerable relevance to the technique of alcohol septal reduction.

(Heart 2000;83:e7) www.heartinl.com/cgi/content/full/83/4/e7 